\title{
Search for gamma-ray emission from AGNs with ultra-fast outflows as candidate cosmic-ray accelerators
}

\author{
Yayoi Tomono*1, Masaaki Hayashida ${ }^{2}$ on behalf of the Fermi-LAT collaboration., \\ Susumu Inoue ${ }^{3}$, Junko Kushida ${ }^{1}$, and Kyoshi Nishijima ${ }^{1}$ \\ ${ }^{1}$ Department of Physics, Tokai University, 4-1-1 Kita-Kaname, Hiratsuka, Kanagawa, \\ 259-1292, Japan \\ ${ }^{2}$ Institute for Cosmic Ray Research, University of Tokyo, 5-1-5 Kashiwanoha, Kashiwa, Chiba, \\ 277-8582, Japan \\ ${ }^{3}$ Max-Planck-Institut für Physik, Föhringer Ring 6, 80805 München, Germany \\ E-mail: tomono@tkikam.sp.u-tokai.ac.jp
}

Recent X-ray observations of active galactic nuclei (AGNs) have revealed the widespread existence of ultra-fast outflows (UFOs), i.e. powerful outflows of baryonic material with velocities $\gtrsim 10^{4} \mathrm{~km} \mathrm{~s}^{-1}(\sim 0.033 \mathrm{c})$, seen as variable, blueshifted absorption features of ionized heavy elements. They have been interpreted as winds driven by the accretion disk and may be responsible for feedback onto their host galaxies, but the physical mechanisms at work are still unknown. In such outflows, various types of shocks are likely to form, either external shocks due to interaction with the ambient medium, or internal shocks due to inhomogeneities within the flow. Such shocks can accelerate electrons and protons to high energies and potentially induce nonthermal emission in various wavebands. In this context, we search for gamma-ray emission from AGNs with known UFOs, using Fermi-LAT data $>100 \mathrm{MeV}$ spanning more than 6 years. The AGN sample of Tombesi et al 2010 is used, with 42 radio-quiet AGNs listed as UFO candidates based on a systematic search for blueshifted Fe $\mathrm{K}$ absorption lines. In our current analysis, no significant gamma-ray excess is found from any objects in the sample. We compute $95 \%$ confidence level gamma-ray upper limits (ULs) for all analyzed sources, yielding a mean value for the integrated photon flux ( $\geq 100 \mathrm{MeV}$ ) UL of $\sim 3 \times 10^{-9}$ photons $\mathrm{cm}^{-2} \mathrm{~s}^{-1}$, and in the range of $10^{40}-10^{44} \mathrm{erg} \mathrm{s}^{-1}$ for ULs on the gamma-ray luminosity $(100 \mathrm{MeV}-100 \mathrm{GeV})$. To assess the properties of this sample with fast outflows, we systematically compare these results with infrared and radio observations, as well as the estimated kinetic power of the outflow. Our Fermi-LAT ULs can constrain the ratio of gamma-ray luminosity to outflow kinetic power down to values as low as 0.0001 . These results provide unique constraints, not only on nonthermal processes in such outflows, but also on the physical conditions therein, such as magnetic fields.

The 34th International Cosmic Ray Conference,

30 July- 6 August, 2015

The Hague, The Netherlands

${ }^{*}$ Speaker. 


\section{Introduction}

Powerful outflows of baryonic material with velocities $\gtrsim 10^{4} \mathrm{~km} \mathrm{~s}^{-1}(v \gtrsim 0.033 c)$ have been detected in recent X-ray observations of active galactic nuclei (AGNs) as variable, blueshifted Fe $\mathrm{K}$ absorption features [4 10]. They are sometimes called ultra-fast outflows (UFOs) and observed in radio-quiet (e.g., [18]) as well as radio-loud AGNs (e.g., [19]). They have been interpreted as winds driven by the accretion disk, and may be responsible for feedback onto their host galaxies [7 10]. However, many basic physical aspects, particularly the mechanisms for their formation and acceleration, are still poorly understood [4 22]. In such outflows, various types of shocks are likely to form, either external shocks due to interaction with the ambient medium [14], or internal shocks due to inhomogeneities within the flow [9]. Such shocks can accelerate electrons and protons to high energies and potentially induce nonthermal emission in various wavebands, offering unique information about the physical conditions therein. In this context, we search for gamma-ray emission from AGNs with such outflows, focusing on radio-quiet objects, and also study their radio and infra-red properties.

\section{Source sample selection}

We perform the search for 42 radio-quiet AGNs listed as UFO candidates in [18], together with the luminous objects PDS 456 and IRAS F11119+3257. In this work, we select only radioquiet AGNs to avoid possible confusion with gamma-ray emission from relativistic jets. UFOs have been defined as highly ionized Fe K absorbers with blueshifted velocity $\geq 0.033 c$ [18]. They detected the blueshifted Fe $\mathrm{K}$ absorption features from 19 sources among the 42 radio-quiet AGNs in their list, of which 15 out of 19 sources show velocities greater than $0.033 c$. PDS 456 and IRAS F11119+3257 were observed by the XMM-Newton and the Suzaku X-ray satellites. Fe K absorption features were detected and outflow velocities estimated to be $\sim 0.3 c$ for both objects [13, 22]. In total, these 44 radio-quiet AGNs are included in our list to search for the gamma-ray emission.

\section{Observations and Results}

We search for gamma-ray emission from these 44 AGNs, using Fermi-LAT data in the energy range $0.1-100 \mathrm{GeV}$, spanning more than 6 years between 2008 August 4 and 2014 September 30. We use the standard LAT analysis software, Fermi Science Tools v9r33p0 and the P7REPSOURCE_V15 instrument response functions. Events are extracted within a $15^{\circ} \times 15^{\circ}$ region of interest (ROI) centered on each target object. Gamma-ray fluxes are determined by the binned maximum likelihood fitting using gtlike. Our background model includes LAT 4-year Point Source Catalog (3FGL) [17 within $15^{\circ}$ from the sample position, and Galactic and isotropic diffuse emission, gll_iem_v05_rev1.fit and iso_source_v05.txt, respectively.

We assume point sources with simple power-law models for our targets. The significance of gamma-ray signals from the targets are based on the likelihood ratio tests [11] with test statistics (TS). If no significant gamma-ray excess is found, we derive a 95\% confidence level (CL) upper limit (UL) for the integrated photon flux above $100 \mathrm{MeV}$ with an assumed photon index of $2.5 \mathrm{using}$ Bayesian methods. $\Gamma=2.5$ corresponds to the average photon index for the flux-limited sample of 
"flat spectrum radio quasars" in the 1st Fermi-LAT AGN catalog [1]. The distances are taken from [16, 23] for the targets whose redshifts are below 0.01 except for ESO 383-35 $(z=0.00775)$ and ESO 434-40 $(z=0.00849)$. Otherwise, the luminosity distances are calculated using individual redshift values with $H_{o}=69.6 \mathrm{~km} \mathrm{~s}^{-1} \mathrm{Mpc}^{-1}, \Omega_{\text {matter }}=0.286, \Omega_{\text {vacuum }}=0.714$.

In the current analysis, no significant gamma-ray excess is found from any objects in our AGN sample. We exclude Mrk 841 from our results because of significant contamination by gammaray emission from a nearby bright source, PKS 1502+106 (3FGL J1504.4+1029), located at $0.11^{\circ}$ away from Mrk 841. We note that ESO 323-77, for which a fast outflow was reported in [18], showed a positionally coincident gamma-ray excess during a previous search in the Fermi data for radio-quiet Seyferts. However, the latest LAT catalog (3FGL) reveals that ESO 323-77 lies outside the $95 \%$ containment radius of the nearest Fermi source, 3FGL J1306.8-4031.

The highest TS values among our results are NGC $4151(\mathrm{TS}=8.4)$ and NGC $4593(\mathrm{TS}=8.2)$. Overall, 27/43 (63\%) objects in our sample have TS $<1$ and 35/43 (81\%) have TS $<4$. Figure 11 shows the distribution of resulting ULs for integrated photon fluxes above $100 \mathrm{MeV}$. We compare the UL distribution of 120 radio-quiet Seyferts with previous work using 3 years LAT data. Medians of resulting ULs for integrated photon fluxes above $100 \mathrm{MeV}$ are improved from $\sim 4 \times 10^{-9}$ photons $\mathrm{cm}^{-2} \mathrm{~s}^{-1}$ in the previous work to $\sim 3 \times 10^{-9}$ photons $\mathrm{cm}^{-2} \mathrm{~s}^{-1}$ here. In terms of luminosity between $100 \mathrm{MeV}$ and $100 \mathrm{GeV}$, the ULs correspond to the range of $10^{40}-10^{44} \mathrm{erg} \mathrm{s}^{-1}$.

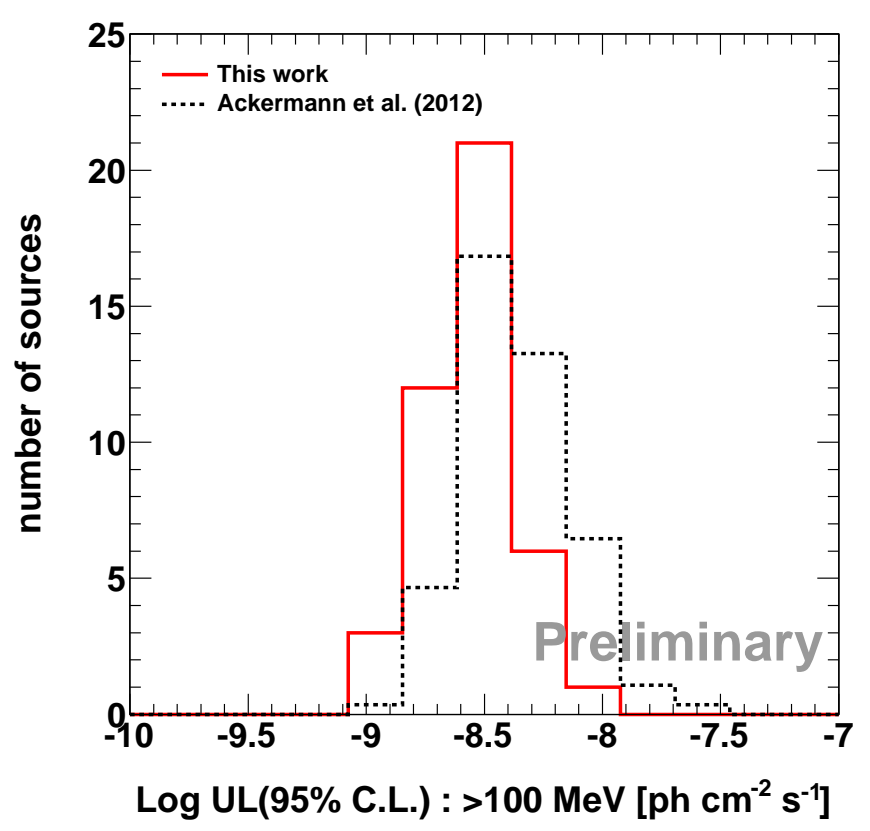

Figure 1: Distribution of the Fermi-LAT photon flux ULs (95\% confidence level) for the analyzed sample of radio-quiet AGNs from this work (solid red line) and 120 radio-quiet Seyferts from [2] (dotted black line). The histogram from 120 radio-quiet Seyferts is scaled by 44/120 to have the same area for ease of comparison. 


\section{Discussion}

To systematically compare these results to infra-red and radio observations, we construct luminosity-luminosity plots between the gamma-ray band and the radio or infra-red bands. The radio luminosities $L_{\mathrm{R}}$ are calculated using monochromatic radio $(1.4 \mathrm{GHz})$ flux density obtained from [6]. If the source is not in this catalog, the $1.4 \mathrm{GHz}$ flux density of the source is calculated by extrapolating or interpolating flux densities in neighboring frequencies given in [5, 12, 24, 25].

The $8-1000 \mu \mathrm{m}$ infra-red luminosities $L_{\mathrm{IR}}$ are calculated using a formula in [15] from four IRAS bands. In Figure 2 we present luminosity-luminosity plots for the radio (left) or the infrared (right) luminosity versus the gamma-ray luminosity UL, $L_{\gamma}$, UL. We see that $L_{\mathrm{R}}$ is distributed in the range of $10^{36}-10^{42} \mathrm{erg} \mathrm{s}^{-1}$ while that for $L_{\mathrm{IR}}$ is $10^{43}-10^{47} \mathrm{erg} \mathrm{s}^{-1}$. The known correlations between the radio, infra-red and gamma-ray luminosities for star-forming and local galaxies 《3] are also indicated in the Figure. Our results of the gamma-ray luminosity ULs are on average more than an order of magnitude higher than the gamma-ray luminosity expected from the correlations. Although only ULs, the data points in the $L_{\gamma}$, UL versus $L_{\mathrm{R}}$ plots are apparently more widely scattered than ones in the $L_{\gamma}$, UL versus $L_{\mathrm{IR}}$.
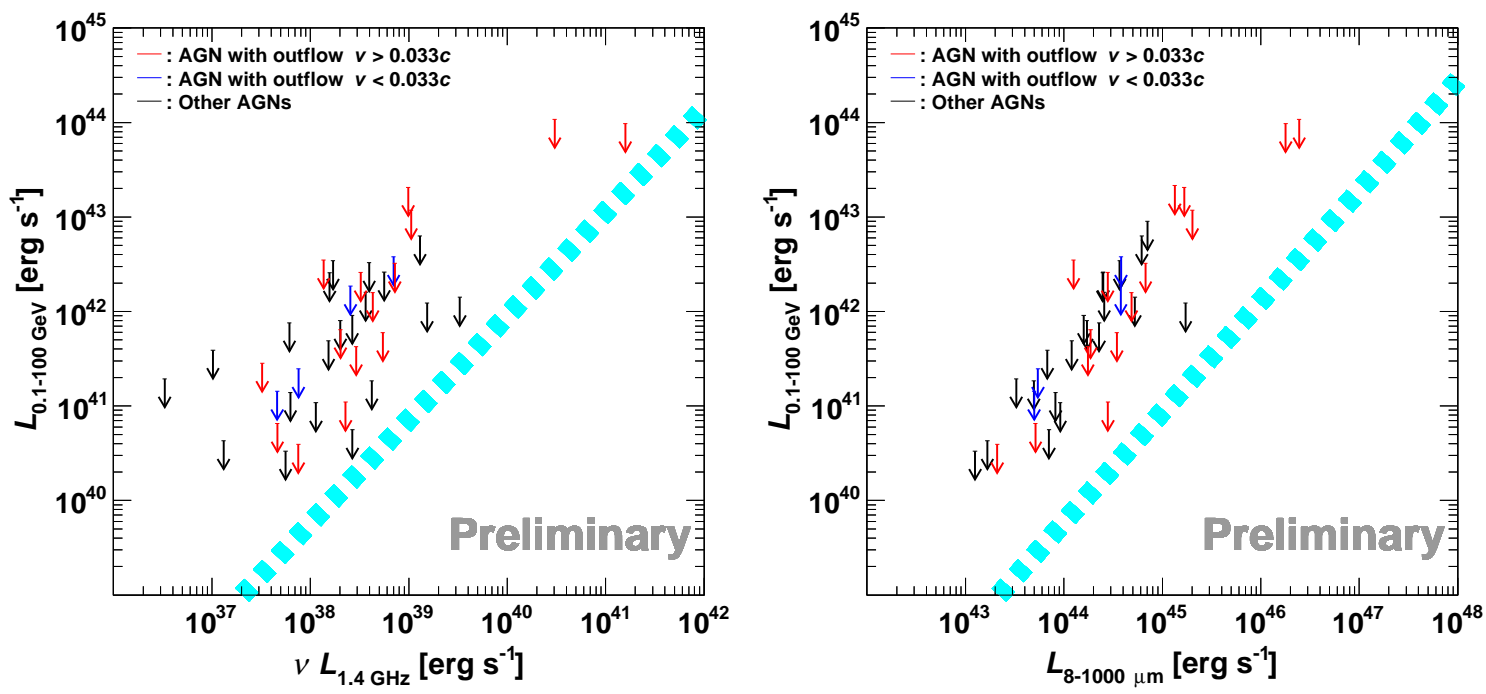

Figure 2: Radio (1.4 GHz; right) and infra-red (8-1000 $\mu \mathrm{m}$; left) luminosities vs. ULs for the gamma-ray $(0.1-100 \mathrm{GeV})$ luminosities for the analyzed sample of radio-quiet AGNs assuming a photon index of 2.5 and $95 \%$ confidence level. Those with outflow velocities greater or less than $10^{4} \mathrm{~km} \mathrm{~s}^{-1}(v \geq 0.033 c)$ are shown as red or blue arrows, respectively. The other AGN samples without detection of the blue-shifted FeK absorption lines are shown in black arrows. The dot cyan line represents the best-fit power-law relation between the radio (or infra-red) and gamma-ray luminosities for star-forming and local galaxies discussed in 3 .

To assess these results in relation to basic properties of the fast outflow, in Figures 3 and 4 , we compare the gamma-ray luminosity UL with the mass outflow rates $\dot{M}$ (minimum or maximum; $\dot{M}_{\text {out min }}$ or $\dot{M}_{\text {out max }}$ ) and the outflow kinetic power $\dot{E}=1 / 2 \dot{M} v^{2}$ (minimum or maximum; $\dot{E}_{\mathrm{K} \min }$ or $\dot{E}_{\mathrm{K} \max }$ ) that were derived from X-ray observations in [20, 13, 22]. The range of $\dot{M}$ and $\dot{E}$ primarily results from large uncertainties in the location of the X-ray absorbers, which range over $\sim 0.0003-0.03 \mathrm{pc}$ for the objects with $v \geq 0.033 c$, and $\sim 0.03-0.3 \mathrm{pc}$ for those with 
$v<0.033 c$ [20]. Note that recent NuSTAR observations of PG 1211+143, the object with the largest $\log \dot{E}\left(=46.9 \pm 0.1 \mathrm{erg} \mathrm{s}^{-1}\right)$, showed no evidence of UFOs at $90 \%$ confidence [26], although this could be a consequence of variability of the absorption features. Our gamma-ray ULs can constrain the ratio of gamma-ray luminosity to the maximum outflow kinetic power down to values as low as 0.0001 .

If inverse Compton cooling is the dominant energy loss mechanism for accelerated electrons, the non-thermal inverse Compton emission may arise in the gamma-ray band, whose luminosity can be described as follow [14].

$$
L_{\gamma} \approx 2.0 \times 10^{-4} \frac{\xi}{0.01} \dot{E}
$$

$\xi$ is fraction of outflow kinetic power which supply to relativistic electrons at the shock. In the maximum outflow kinetic power, Mrk $1095\left(L_{\gamma, \mathrm{UL}} / \dot{E}_{\mathrm{K} \max }=1.0 \times 10^{-4}\right)$ and NGC $4051\left(1.0 \times 10^{-4}\right)$, NGC $7582\left(1.4 \times 10^{-4}\right)$ show lower ratios of $L_{\gamma, \mathrm{UL}} / \dot{E}_{\mathrm{K} \max }$ than the value expected from Eq. 4.1 , PG $1211+143\left(2.6 \times 10^{-4}\right)$ show a slightly higher than the ratio from Eq. 4.1 On the other hand, for the case of the minimum outflow kinetic power, the lowest ratio can be seen in NGC 7582 $\left(4.4 \times 10^{-3}\right)$, which is still much higher than the ratio from Eq. 4.1
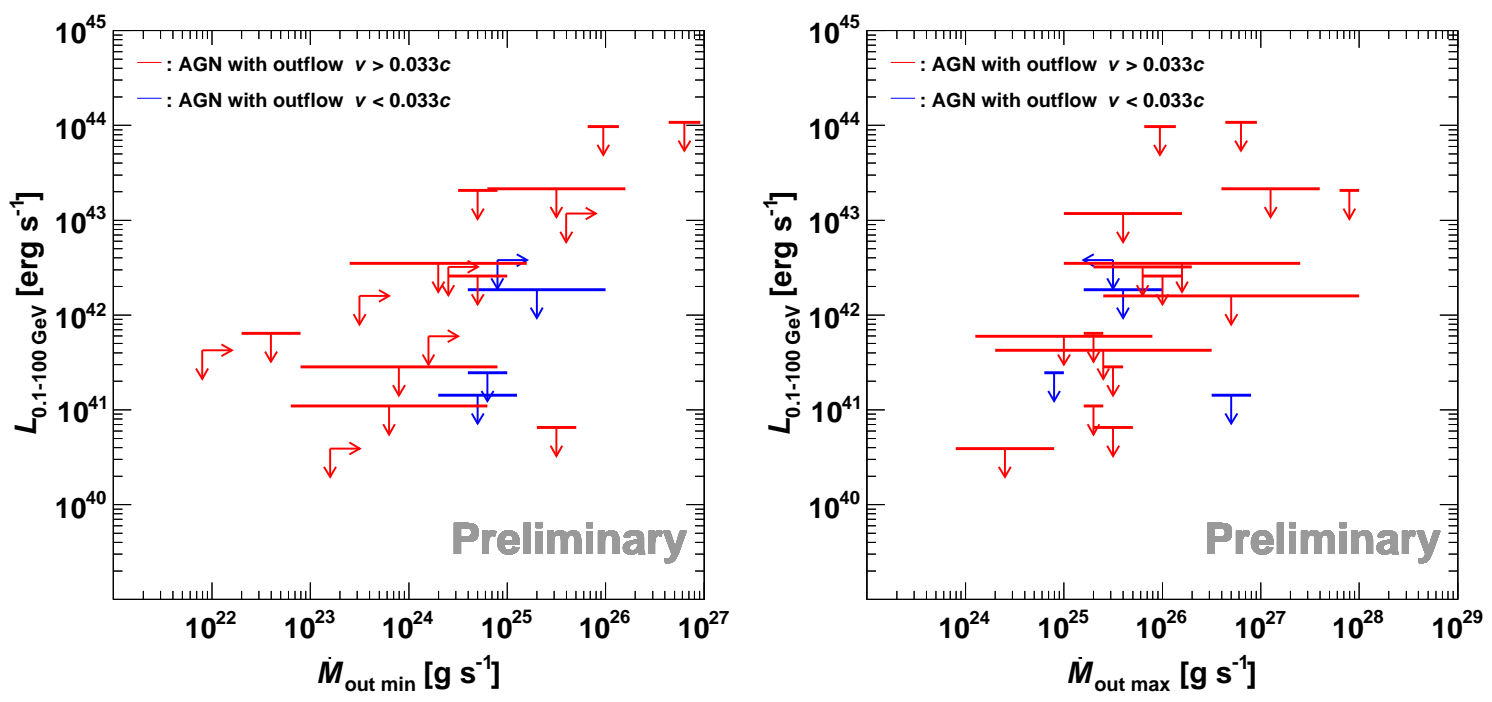

Figure 3: The minimum mass outflow rate (left) and the maximum mass outflow rate (right) vs. ULs for the gamma-ray $(0.1-100 \mathrm{GeV})$ luminosities. We take the mass outflow rates from [13, 20, 22]. Those with outflow velocities greater or less than $10^{4} \mathrm{~km} \mathrm{~s}^{-1}(v>0.033 c)$ are shown as red or blue arrows respectively. The horizontal bars denote uncertainties of the rates.

We construct broadband spectral energy distributions (SEDs) in terms of energy flux (left) and luminosity (right) for PDS 456 (green), PG 1211+143 (magenta) and NGC 4151 (cyan). Those objects have fast outflows of $v \geq 0.033 \mathrm{c}$. The gamma-ray ULs were derived in six energy bins in the range between $100 \mathrm{MeV}$ and $100 \mathrm{GeV}$ in logarithmic scale. In our sample with fast outflows, PDS 456 and PG 1211+143 are two of the most luminous objects, while NGC 4151 is the brightest $\mathrm{X}$-ray source.

Although our gamma-ray search has so far yielded only upper limits, these can nevertheless offer some unique and interesting constraints on the physical properties of AGN fast outflows, 

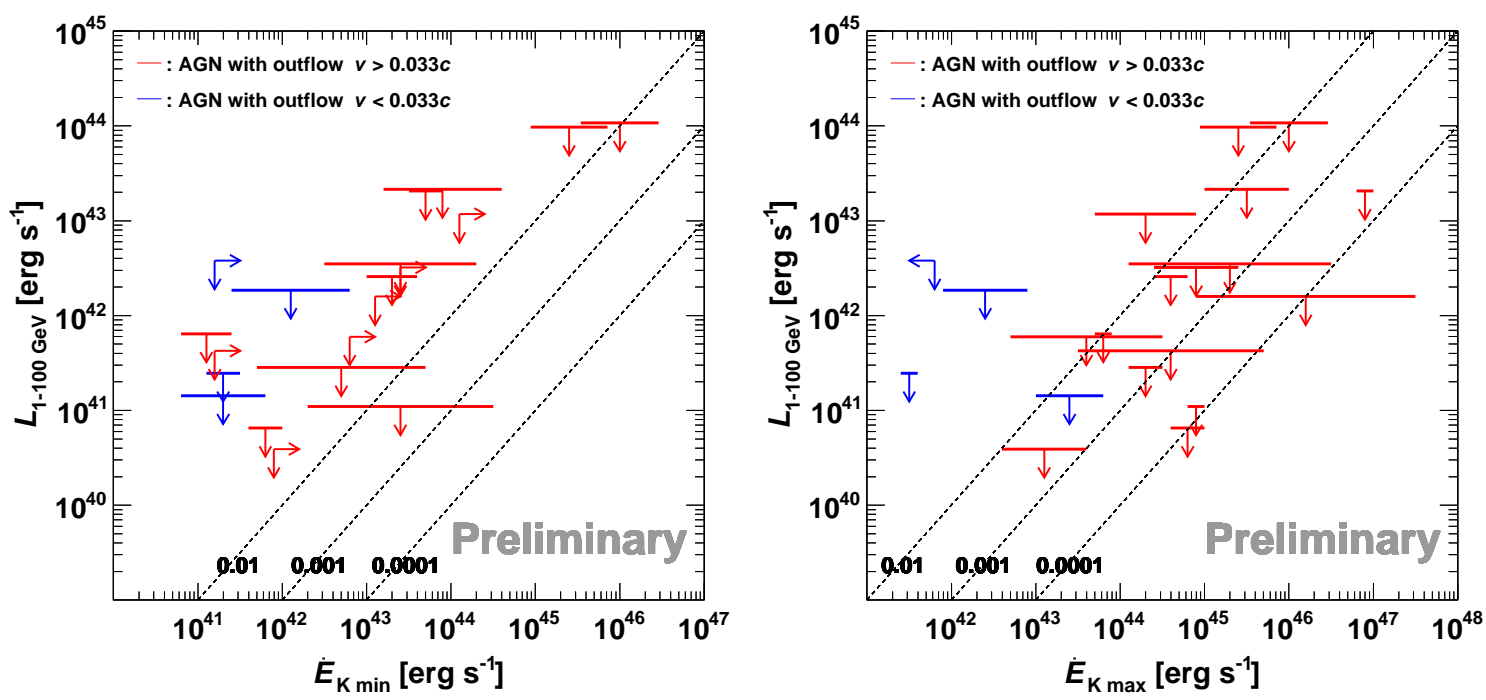

Figure 4: The minimum outflow kinetic power (left) and the maximum outflow kinetic power (right) vs. ULs for the gamma-ray $(0.1-100 \mathrm{GeV})$ luminosities. We took the outflow kinetic power from [13, 20, 22]. Those with outflow velocity greater or less than $10^{4} \mathrm{~km} \mathrm{~s}^{-1}(v>0.033 c)$ are shown as red or blue arrows respectively. The horizontal bars denote uncertainties of the rates. Those plots show Dotted lines from top left to bottom right denote the ratios between the gamma-ray luminosity and the outflow kinetic power 0.01 , 0.001 , and 0.0001 , respectively.
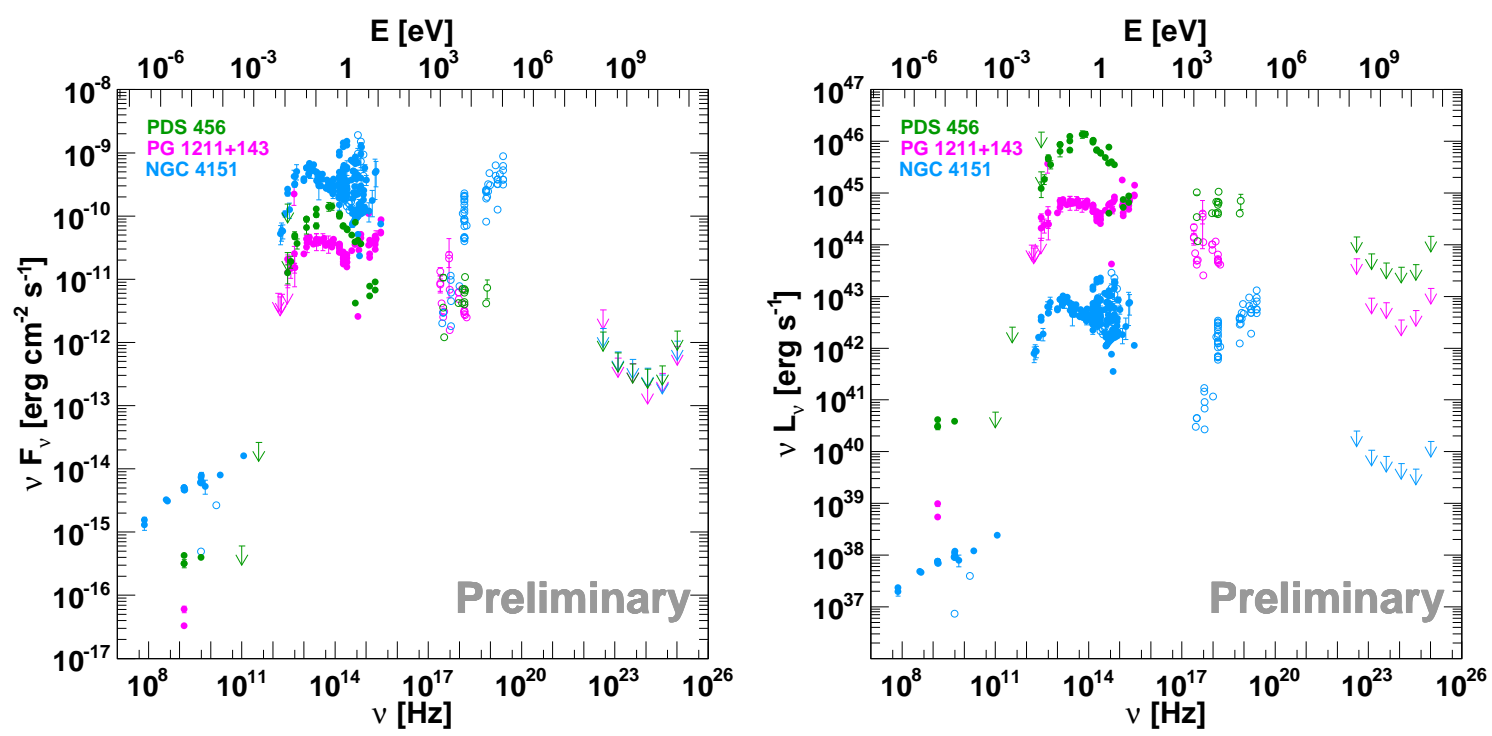

Figure 5: Broadband spectral energy distributions in terms of energy flux (left) and luminosity (right) for PDS 456 (green), PG 1211+143 (magenta) and NGC 4151 (cyan). Gamma-ray ULs $(0.1-100 \mathrm{GeV})$ are derived here while data at other wavelengths are taken from NED. The total flux from the galaxy are marked as full circles, while that from the core region only are marked as open circles. The core fluxes are referred to as "core" or "nuclear" flux in the optical and radio bands, all X-ray results, and VLBI radio observations. The hard X-ray emission is likely predominantly of accretion disk origin. 
particularly the magnetic field strength $B$, under the assumption that their observed radio flux is primarily due to synchrotron emission from electrons accelerated at plausible sites in these outflows. Such electrons can coproduce IC gamma-ray emission, and the ratio of powers for each process radiated by electrons of a given energy is $L_{\mathrm{syn}} / L_{\mathrm{IC}} \simeq U_{\mathrm{B}} / U_{\mathrm{ph}}$, where $U_{\mathrm{B}}=B^{2} / 8 \pi$ and $U_{\mathrm{ph}}$ are the energy densities of the magnetic field and the soft photons for IC upscattering, respectively, and Klein-Nishina effects are neglected. The latter is likely dominated by direct and/or reprocessed photons from the nucleus for the objects of our interest, the details depending on the exact location of the acceleration site, etc. 9]. For simplicity, here we assume that these are primarily photons from within the broad line region with typical frequency $v_{\mathrm{BLR}}=2.4 \times 10^{15} \mathrm{~Hz}(\sim 10 \mathrm{eV})$ and energy density $U_{\mathrm{BLR}}=2.7 \times 10^{-2} \mathrm{erg} \mathrm{cm}^{-3}$ [8]. For electrons of a given energy, the ratio of the typical frequencies of IC and synchrotron emission is $v_{\mathrm{IC}} / v_{\mathrm{syn}}=2 \times 10^{12}(B / \mathrm{mG})$, so that those producing e.g. $\sim 1 \mathrm{GeV}$ gamma rays also radiate at $\sim 100 \mathrm{GHz}(B / \mathrm{mG})$. Although the synchrotron flux at the latter frequency is generally overwhelmed by thermal components and not directly observable, it may be inferred by extrapolating the measured $1.4 \mathrm{GHz}$ flux. For example, taking $L_{\mathrm{syn}} / L_{\mathrm{IC}} \sim 10^{-2}$, we can deduce $B>0.1 \mathrm{G}$ for the outflows in NGC 4151. While these constraints are not strong enough to discriminate physical models for the formation of AGN winds, e.g. whether they are radiation-driven of magnetically-driven [4 21], they offer a tantalizing glimpse into the physical conditions of AGN winds that are not possible from X-ray or optical/UV observations alone. Note that except for NGC 4151 and a few other well-observed objects, observed radio fluxes are likely contaminated by contributions from the host galaxy so that future radio observations with high sensitivity and spatial resolution are desirable.

\section{Acknowledgement}

The Fermi-LAT Collaboration acknowledges support from a number of agencies and institutes for both development and the operation of the LAT as well as scientific data analysis. These include NASA and DOE in the United States, CEA/Irfu and IN2P3/CNRS in France, ASI and INFN in Italy, MEXT, KEK, and JAXA in Japan, and the K. A. Wallenberg Foundation, the Swedish Research Council and the National Space Board in Sweden. Additional support from INAF in Italy and CNES in France for science analysis during the operations phase is also gratefully acknowledged.

\section{References}

[1] Abdo, A. A., Ackermann, M., Ajello, M., et al. 2010, ApJ, 715, 429

[2] Ackermann, M., Ajello, M., Allafort, A., et al. 2012, ApJ, 747, 104

[3] Ackermann, M., Ajello, M., Allafort, A., et al. 2012, ApJ, 755, 164

[4] Cappi, M., Done, C., Behar, F. et al. arXiv:1306.2330

[5] Condon, J. J., Helou, G., Sanders, D. B., \& Soifer, B. T. 1996, ApJS, 103, 81

[6] Condon, J. J., Cotton, W. D., Greisen, E. W., et al. 1998, AJ, 115, 1693

[7] Fabian, A. C. 2012, ARA\&A, 50, 455 
[8] Ghisellini, G., Tavecchio, F. 2009, MNRAS, 397, 985

[9] Inoue, S., Liu, R.-Y., Murase, K. 2015, PoS (ICRC2015) 925

[10] King, A. \& Pounds, K. arXiv:1503.05206

[11] Mattox, J. R., Bertsch, D. L., Chiang, J., et al. 1996, ApJ, 461, 396

[12] Mauch, T., Murphy, T., Buttery, H. J., et al. 2008, VizieR Online Data Catalog, 8081, 0

[13] Nardini, E., Reeves, J. N., Gofford, J., et al. 2015, Science, 347, 860

[14] Nims, J., Quataert, E., \& Faucher-Giguère, C.-A. 2015, MNRAS, 447, 3612

[15] Sanders, D. B., \& Mirabel, I. F. 1996, ARA\&A, 34, 749

[16] Sanders, D. B., Mazzarella, J. M., Kim, D.-C., Surace, J. A., \& Soifer, B. T. 2003, AJ, 126, 1607

[17] The Fermi-LAT Collaboration 2015, arXiv:1501.02003

[18] Tombesi, F., Cappi, M., Reeves, J. N., et al. 2010, A\&A, 521, A57

[19] Tombesi, F., Sambruna, R. M., Reeves, J. N., et al. 2010, ApJ, 719, 700

[20] Tombesi, F., Cappi, M., Reeves, J. N., \& Braito, V. 2012, MNRAS, 422, L1

[21] Tombesi, F., Cappi, M., Reeves, J. N., et al. 2013, MNRAS, 430, 1102

[22] Tombesi, F., Meléndez, M., Veilleux, S., et al. 2015, Nature, 519, 436

[23] Tully, R. B., Rizzi, L., Shaya, E. J., et al. 2009, AJ, 138, 323

[24] Ulvestad, J. S., \& Wilson, A. S. 1984, ApJ, 285, 439

[25] Wright, A., \& Otrupcek, R. 1990, PKS Catalog (1990), 0

[26] Zoghbi, A., Miller, J. M., Walton, D. J., et al. 2015, ApJ Letters, 799, L24 